\title{
Las comunidades y sus revistas científicas
}

\section{The scientific communities and their journals}

\section{Leonardo Romero}

Editor Jefe, Instituto de Investigación de Ciencias Biológicas Antonio Raimondi, Facultad de Ciencias Biológicas, Universidad Nacional Mayor de San Marcos. Apartado 11-0058, Lima 11, Perú. Email: Iromeroc@unmsm.edu.pe

Las investigaciones realizadas y expuestas ante las sociedades científicas, tuvieron en las revistas científicas un medio ex professo de comunicación y diseminación de sus memorias y descubrimientos. Sin embargo en el escenario actual los investigadores y las revistas científicas adoptan nuevas características y se ven influenciadas por diferentes tendencias que requieren una estrategia de desarrollo editorial adecuada.

\section{Comprender a las revistas científicas}

Podemos encontrar que no existe una definición propia de revista científica, aunque lo más común y divulgado sea una definición operacional, descriptiva, procedente de la actividad documentalista y eminentemente práctica la cual refiere a la periodicidad, los artículos científicos/técnicos que son publicados, la originalidad y su sistema de peer review.

Por otro lado, cada revista se particulariza y define en sus propósitos, especializaciones, y estilos creando un panorama muy variado de revistas científicas. Aunque en común, la mayoría de las revistas incluyen el propósito de solamente aceptar artículos de calidad y editados con los más altos estándares.

El nacimiento, historia, sobrevivencia y trayectoria de cada revista son factores que tal vez nos permiten apreciar mejor este escenario. Las revistas científicas han aparecido y desaparecido desde el siglo XVII, pero, una información importante es que la aparición de revistas científicas tiene una tasa casi constante de 3,6\% anual desde el siglo XIX, un ritmo que aparentemente depende del incremento constante del número de investigadores y por lo tanto el número de artículos y por consiguientela demanda por nuevas revistas ${ }^{1,2}$.

Todas las revistas científicas no tienen la misma procedencia, las primeras fueron establecidas por sociedades científicas (v.g. The Proceedings of the Royal Society), las academias, generalmente en relación directa con los gobiernos también generaron importantes revistas (Comptes rendus de l'Académie des sciences). Instituciones académicas como universidades e institutos de investigación también generaron revistas. Las diferencias entre ellas fueron los detalles de sus objetivos y finalidades.

El escenario cambió después de la segunda guerra mundial cuando las grandes editoras comenzaron a desarrollarse y unas pocas se convierten en las casas editoras de las revistas de sociedades científicas, institutos y universidades. Los servicios de esas editoras incluían el proceso editorial, el peer review y la distribución, los cuales se hacen más eficientes y competitivos. En el año 2006 se calculó en más de 20000 las casas editoras, de ellas 657 producían 11500 títulos. El 64\% del volumen de los artículos era editado por casas editoras comerciales (con fines de lucro), el $30 \%$ por sociedades científicas, el $4 \%$ por universidades y el $2 \%$ por otros ${ }^{3}$. Las grandes editoras, como en cualquier gran negocio tienen un desarrollo muy dinámico, fusionándose y creciendo, por ejemplo en el 2007 se fusionaron Blackwell Publishing con Wiley's Global Scientific acumulando 1400 revistas, todas indexadas en la base de datos ISI.

Al escenario reciente debemos sumar el impacto de la INTERNET. A diferencia de un libro o un magazín general, una de las características del uso de las revistas científicas es que no son leídos todos los artículos. Los investigadores buscan los más cercanos o relacionados a sus investigaciones; lo que hace que cualquier revista científica por más especializada que sea, siempre tendrá algún grado de generalidad dentro del ámbito de un investigador. La INTERNET ha exacerbado esta característica, la búsqueda de los archivos se lleva a cabo en varias revistas a la vez, muchas veces de diferentes casas editoras. Los servicios de indización facilitan este modelo de búsqueda, por ejemplo: ScienceDirect ofrece 2500 revistas y más de 6000 libros online; Blackwell Synergy — Wiley InterScience ofrecen 1400 revistas, 6000 libros online y SpringerLink ofrece más de 1250 revistas y más de 10000 libros online.

Podríamos concluir que una revista científica por más que cuente con un soporte de investigadores que aporten sus trabajos para ser publicados y un amplio mercado de lectores, necesitará más infraestructura para sobrevivir en esta competencia que demanda mayor visibilidad y circulación. En este contexto la inclusión e indización de una revista en una base de datos le proporciona mejores condiciones para sobrevivir.

\section{El impacto de la bibliometricomania}

Bajo el planteamiento que unas cuantas revistas científicas cubrían un porcentaje muy significativo de la producción científica mundial, se llegó al corolario que existían demasiadas revistas y que habría que concentrar la información en el menor número posible, seleccionar unas cuantas que condujeran y constituyeran el mainstream académico. Sólo algunas editoras e instituciones pudieron llegar a este nivel, y que ahora son consideradas el horizonte de calidad al que debe llegar toda revista científica. Todo en el nombre del llamado Factor de Impacto.

En un primer momento el Factor de Impacto fue usado para racionalizar las adquisiciones y colecciones de revistas en las grandes bibliotecas, una forma de dirigir la inversión de los institutos, pero en la actualidad, en el primer mundo, los investigadores presentan su currículo incluyendo el Factor de Impacto de sus trabajos y el de las revistas en que publican; las instituciones también presentan el Factor de Impacto como un indicador de productividad y prestigio, los países miden sus avances en ciencia y tecnología de la misma manera y por consiguiente los fondos 
para investigación se dirigen a los que muestran un mejor Factor de Impacto. Esto nos muestra un sistema coherente que dirige de manera fácil la investigación. Aunque es una practica es común en los paises del primer mundo, no son extraños en los más cercanos a nosotros; como así nos narra Anna Maria Prat ${ }^{4}$ sobre el uso de la bibliometría en la vida de las revistas chilenas y también en los presupuestos universitarios. Una perspectiva muy difundida es que en el mundo existe un exagerado interés por la evaluación bibliométrica de las revistas científicas, lo cual en realidad es tiene como trasfondo la competencia por lograr mayores fondos, al considerar que las publicaciones son productos que miden el grado de cumplimiento dentro de la concepción política de Investigación-Desarrollo-Innovación $(\mathrm{I}+\mathrm{D}+\mathrm{i})^{5}$. Es probable que esta concepción pueda desdoblare en otros componentes o formas de resolver el problema (ciencia-sociedad-economía y educación-investigación-innovación) y por lo tanto existan otros productos que medir. Reflexionando podriamos decir que el problema de trasfondo es como hacer más rentable la ciencia para la "sociedad", como acelerar la recepción del beneficio que "debe brindar" la ciencia, y como poner en práctica las soluciones "científicas" a los problemas sociales y económicos.

El impacto de toda esta tendencia es un feedback elemental, los investigadores eligen las revistas en las que presentan sus artículos en función de "obtener un mayor puntaje"; es decir clasifican jerárquicamente a las revistas, así como también a sus resultados. Tendencia que la Rev peru biol sufre en carne propia; tendencia en la que se propicia el absurdo de publicar en una revista que nunca será leída por esa sociedad que sí podría salir beneficiada de ese conocimiento, tendencia y realidad que la paradigmática política $\mathrm{I}+\mathrm{D}+\mathrm{i}$ no toma en cuenta.

\section{Pensando como sobrevivir}

Modestamente la Rev peru biol incrementó el promedio de artículos por número desde 9,7 en el periodo 1998-2002; hasta 19,3 artículos en el periodo 2003-2007; lo que significó un incremento de 199\%. En la actualidad el promedio mensual de artículos recibidos es de 7,3, y el número de artículos rechazados en promedio es del $45 \%$ de los recibidos. Para el periodo 1998-2007, el 21,4\% de primeros autores fueron docentes de la Facultad de Ciencias Biológicas de la UNMSM, el 61,9\% pertenecieron a otras instituciones, y el 16,7\% fueron tesistas. El tema más importante tratado en la Rev peru biol es la biodiversidad en sus diferentes aspectos, destacando la descripción de nuevas especies.

Aunque esta información pueda ser alentadora, es insuficiente ya que aun no se ha logrado consolidar la comunidad científica que utiliza la revista, como medio de difusión o como lector que utiliza la información de sus páginas. Solamente se cuenta con un repositorio de los artículos, faltando implementar procesos automatizados de edición como los ofrecidos por el Open Journal Systems, que harían más veloz y transparente el proceso de edición y revisión. La cobertura internacional demanda más inversión para lograr los envíos de postales de la versión impresa.
Recibir, procesar y distribuir artículos no son las únicas condiciones para catalogar a una revista de competitiva. Se exige un desarrollo sostenido, con incrementos sostenidos de artículos y más inversión económica, sin ambos no podremos considerar el éxito. ¿Qué implica incrementar el numero de artículos?, encandilar a los autores, es decir tener un factor de impacto (= estar en Journal Citation Index), tener una reputación dentro de la comunidad a la que va dirigida la revista, tener un cuerpo editorial profesional, procesos de publicación y edición rápidos, cobertura internacional, relaciones con sociedades científicas, tecnología (comunicación, distribución y edición por INTERNET), entre otras cosas ${ }^{6}$.

El nacimiento de la Rev peru biol sucedió en 1974 bajo el entusiasmo de la Asociación de Biólogos de la Universidad de San Marcos, sin embargo esta primera etapa fue muy difícil, reactivándose en 1998 cuando la Facultad de Ciencias Biológicas de la Universidad Nacional Mayor de San Marcos se convirtió en la casa editora. El núcleo de la comunidad científica que sustenta en la actualidad la Rev peru biol son los miembros del Instituto de Investigación de Ciencias Biológicas Antonio Raimondi, los cuales son insuficientes para alimentar con su producción científica la cantidad de artículos necesarios para lograr el desarrollo sostenido de la revista. Todo lo que sucede inmediatamente y percibimos cercanamente, es sólo parte de una realidad más compleja en la cual encontramos ciertos indicios de nuevas posibles situaciones, por ejemplo el incremento de investigadores a los cuales hay que tomar como un factor en el moldeamiento de la tarea editorial futura. Las publicaciones en grupo y el trabajo multidisciplinario, implican otros cambios editoriales y también en las comunidades de lectores e investigadores. Bases de datos más generales y con temáticas emergentes. La adopción de mejores tecnologías de diseminación. En resumen la estrategia de desarrollo editorial tiene que analizar y proponer alternativas a los nuevos tipos de comunidades científicas que se están formando.

1 Mabe M. 2003. The growth and number of journals. Serials 16(2): $191-197$.

2 Mabe M., M. Amin. 2001. Growth dynamics of scholarly and scientific journals. Scientometrics. 51(1): 147-162

3 Mark Ware Consulting Ltd. 2006. Scientific publishing in transition: an overview of current developments. (http://www.stm-assoc. org/storage/Scientific_Publishing_in_Transition_White_Paper.pdf) acceso: 02/011/08

4 Prat A. M. 2001. Evaluación de la producción científica como instrumento para el desarrollo de la ciencia y la tecnología. ACIMED (supl.): 111-114 (http://bvs.sld.cu/revistas/aci/vol9_s_01/ sci16100.pdf) acceso: 02/011/08

5 Pulido San Román A. 2006. Investigación, innovación y universidad en la nueva sociedad del conocimiento. Encuentros Multidisciplinares 22. (http://www.encuentros-multidisciplinares.org/ Revistan $22 /$ Antonio\%20Pulido\%20San\%20Román.pdf) acceso: 02/011/08

6 Thompson, P.J. 2007. How To Choose the right journal for your manuscript. Chest 132: 1073-1076 